\section{Endocrinology and diabetes}

\section{G1 CHILDHOOD CRANIOPHARYNGIOMA: MORBIDITY AND MORTALITY IN MANCHESTER OVER THE LAST 20 YEARS}

D.A. Iles', H.R. Gattamaneni', J. Leggate', J. Thorne', C. West', D.A. Price', P.E. Clayton', C.M. Hall'. 'Royal Manchester Children's Hospital; ${ }^{2}$ The Christie Hospital, Manchester

Background: Childhood craniopharyngioma (CC) is a locally aggressive tumour with significant associated morbidity and the goals of treatment include removal of the tumour, prevention of recurrence, adequate hormonal replacement and minimisation of damage to the hypothalamus and optic tracts. Management strategies vary: neurosurgery may be partial (PS) or complete (C) and postoperative radiotherapy (XRT) may follow PS or be used in the event of tumour recurrence (TR).

Aims: To assess the morbidity and mortality associated with different treatment modalities.

Methods: The casenotes of 25 children diagnosed with CC in Manchester over the last 20 years were reviewed. Comparisons were made between treatment groups: (A) PS and immediate XRT $(n=10)$ versus $C S$ and no XRT $(n=5)$, (B) PS and no XRT $(n=7)$ versus $C S$ and no XRT $(n=5)$ and (C) XRT (n=12) versus surgery alone $(n=13)$. Neurosurgical information was incomplete in 3 subjects. RESULTS: The mean age (sd) at diagnosis was 7.7 (4.5) years and the mean length of follow up was 8.2 (4.3) years. 2 patients died: neither had XRT and both had multiple operations. Treatment modality did not significantly affect the following postoperative markers of morbidity: endocrinopathy (hypopituitarism in 24 of 25), change in BMISDS (0.5 [1.5]), change in height SDS (0.7 [1.3]), visual impairment (present in 17 of 25) or hypothalamic morbidity (recorded in 16 of 25). Treatment modality significantly affected TR: In group (A) tumour recurred in 3 of 5 patients treated with CS alone compared with 0 of 10 treated with PS and XRT ( $p=0.02)$. In group (B), there was no significant difference in TR between PS alone ( 3 of 7) compared to CS alone (3 of 5). In group (C), TR was significantly lower in XRT (0 of 12) versus no XRT (7 of 13) $(p=0.005)$

Conclusions: Our data indicate that immediate postoperative XRT prevents tumour recurrence and suggest that treatment modality does not affect morbidity. However long term prospective neuropsychological follow up will be necessary to confirm these findings.

\section{G2 GROWTH HORMONE (GH) PROVOCATION TESTS AND THE RESPONSE TO GH THERAPY IN CHILDREN WITH GH DEFICIENCY}

T.J.Cole, D.B. Dunger. Centre for Paediatric Epidemiology \& Biostatistics, Institute of Child Health, London WCIN 1EH; Department of Paediatrics, University of Cambridge, Addenbrookes Hospital, Cambridge CB2 2QQ

Aims: to identify factors, particularly the response to the $\mathrm{GH}$ provocation test, that affect the growth response to $\mathrm{GH}$ therapy in children with growth hormone deficiency (GHD).

Methods: data for 513 (72\% male) patients with a diagnosis of GHD were obtained from the Pharmacia KIGS UK database (inclusion criteria: a response of $<20 \mathrm{mU} / \mathrm{l}$ to a $\mathrm{GH}$ provocation test, prepubertal and $<10$ years old at diagnosis, and $1+$ years of GH therapy). Growth response was defined as the change in height SDS (revised UK reference) in each of the first 2 years of treatment, and was adjusted for height SDS 1 year earlier and other significant covariates; $\mathrm{GH}$ provocation test response, $\mathrm{GH}$ dose and frequency, age, weight SDS, birth weight, gestation, and midparent height. Significant covariates were identified using backwards stepwise multiple regression

Results: mean height SDS at baseline was -3.4 (SD 0.9) at mean age 7.8 (SD 1.3) years. Growth response in the $1^{\text {st }}\left(2^{\text {nd }}\right)$ year was $+0.72(+0.37)$ SDS units. Nine factors predicted $1^{\text {st }}$ year response (N $=316)$, in particular $\mathrm{GH}$ provocation test response $(t=-7.5)$, previous height SDS $(t=-5.8)$, midparent height $(t=4.7), \mathrm{GH}$ frequency $(t=$ $4.2)$ and age $(t=-3.3)$. They explained $42 \%$ of variance. In contrast only 3 factors predicted $2^{\text {nd }}$ year response $(N=246)$, primarily $G H$ provocation test response $(t=-3.8)$, explaining $7 \%$ of variance. $1^{\text {st }}$ year growth response, added to the $2^{\text {nd }}$ year growth response model, was not only highly significant $(t=6.8)$ but it also rendered insignificant the $\mathrm{GH}$ provocation test response $(t=0.7)$. This extended model explained $22 \%$ of variance.

Conclusions: the $\mathrm{GH}$ provocation test predicts well the growth response to $\mathrm{GH}$ therapy in the $1^{\text {st }}$ year, but it is less predictive in the $2^{\text {nd }}$ year and leaves considerable variance unexplained, which may relate to the validity of the test for defining GHD. Combining the provocation test with an analysis of $1^{\text {st }}$ year growth response, and then re-testing at 1 year, may be a more effective way of defining GHD. This could be tested with a clinical trial.

\section{G3 IMPROVED FINAL HEIGHT IN TURNER'S SYNDROME FOLLOWING GROWTH PROMOTING TREATMENT AT A SINGLE CENTRE}

E.J.Gault, W.F. Paterson, M.D.C. Donaldson. Department of Child Health, University of Glasgow, Royal Hospital for Sick Children, Glasgow, G3 8SJ

Growth hormone $(\mathrm{GH})$ therapy has been shown to improve final height (FH) in groups of girls with Turner's syndrome (TS). However, an earlier Scottish analysis revealed $\mathrm{FH}$ a mere $0.6 \mathrm{~cm}$ greater than untreated projected adult height $(\mathrm{PAH})(1)$. This study aimed to identify the $\mathrm{FH}$ of girls with TS treated subsequently at a single Scottish centre and compare it with results of the national analysis. Data were available for 27 girls, aged $=15$ years, who have reached $\mathrm{FH}$ and were not included in the earlier analysis. Although not analysed, a further 7 patients were identified whose growth promoting treatment continues and whose current height range is $150.5-155.8 \mathrm{~cm}$.

Abstract G3 Patient, treatment \& outcome data,Glasgow 1994-2001 \& Scotland 1988-1994

\begin{tabular}{|c|c|c|c|}
\hline & $\begin{array}{l}\text { Glasgow } \\
(n=27)\end{array}$ & $\begin{array}{l}\text { Scotland } \\
(n=26)\end{array}$ & $P$ \\
\hline $45, \mathrm{X} /$ other & $11 / 16$ & $12 / 14$ & ns \\
\hline$\% \mathrm{XrX}_{\mathrm{rX}}$ & $3.7 \%$ & $30.8 \%$ & $<0.01$ \\
\hline PAH $(\mathrm{cm})$ & $145.3(5.2)$ & $142.0(6.0)$ & $<0.05$ \\
\hline$M P H(c m)$ & $159.7(5.4)$ & unavailable & - \\
\hline Age at $\mathrm{GH}$ start (yrs) & $9.9(2.7)$ & $12.1(1.7)$ & $<0.01$ \\
\hline Duration of GH (yrs) & $6.3(2.5)$ & $3.7(1.1)$ & $<0.01$ \\
\hline $\mathrm{GH}$ start dose (IU $\left./ \mathrm{m}^{2} / \mathrm{wk}\right)$ & $22.7(4.4)$ & unavailable* & - \\
\hline Oxandrolone (n) & 17 & 15 & ns \\
\hline Age at $E_{2}$ start (yrs) ${ }^{\#}$ & $12.6(1.8)(n=23)$ & $12.8(1.8)(n=21)$ & ns \\
\hline $\mathrm{FH}(\mathrm{cm})$ & $151.0(4.8)$ & $142.6(5.6)$ & $<0.01$ \\
\hline FH-PAH $(\mathrm{cm})$ & $5.7(4.8)$ & $0.6(3.6)$ & $<0.01$ \\
\hline$\Delta \mathrm{TS} H \mathrm{H}$ SDS & $0.9(0.7)$ & $0.2(0.5)$ & $<0.01$ \\
\hline
\end{tabular}

* Mean GH start dose for total sample of 72 from which this cohort of 26 taken $-22.5 \mathrm{IU} / \mathrm{m}^{2} / \mathrm{wk}$

\#Excluded if entered puberty spontaneously \& pharmacological induction not required.

Multiple regression analysis of the Glasgow group identified mid-parental height (MPH), PAH \& GH start dose as statistically significant predictors of $\mathrm{FH}(p<0.05)$. While the Glasgow group was projected to be the taller of the 2 groups, the magnitude of difference is attributable, largely, to the consistent use of $\mathrm{GH}$, from a younger age and, hence, for longer prior to oestrogen induction, by a committed "growth team" at a dedicated clinic.

1. Chu et al. Acta Paediatr 1997;86:160-64.

\section{G4 COGNITIVE FUNCTION IN CHILDREN WITH CONGENITAL HYPOPITUITARISM}

K. Brown', J. Rodgers' , M. Gibson', T.D. Cheetham'. Departments of Child Health' and Clinical Psychology'; Royal Victoria Infirmary and Newcastle University, Queen Victoria Road, Newcastle upon Tyne NE 1 4LP

Aims: There is little objective data on the cognitive function of children with pituitary hormone deficiency (PHD). There is evidence to suggest that there may be unrecognised visual impairment that could impinge on intellectual development. We have therefore undertaken a pilot study to assess cognitive skills in this group of patients.

Methods: 14 children with PHD were assessed by a psychologist using the Wechsler Intelligence Scale for Children (WISC-III UK). This provides verbal, performance and full-scale IQ scores. Three children had isolated growth hormone deficiency with an abnormal MRI and 11 had multiple PHD. Two of the patients had associated visual impairment due to hypoplastic optic nerves. In ten patients data was also collected from a sibling without PHD.

Results: The patient's average full-scale IQ score was 87 (range 45-128). Paired t-test analysis on the ten sibling pairs showed that 
patients had significantly lower full-scale IQ (mean 89 vs 104; $p<0.01$ ) and performance IQ scores (mean 92 vs $115 ; p<0.002$ ) when compared to their sibling control. Verbal skills were not significantly different $(90$ vs $94 ; p=0.4)$. The difference in performance $I Q$ can be accounted for by the patients' poorer performance on tasks assessing perceptual organisational skills $(p<0.01)$. This difference was independent of the presence of recognised visual impairment.

Conclusions: Children with PHD perform less well than their siblings. Whether this is linked to illness such as hypoglycaemia in early life or is a manifestation of an underlying brain malformation is unclear. The impaired perceptual organisational ability may be linked to an unrecognised visual processing deficit. These data are of value when counselling parents and planning a child's care and education.

\section{G5 PITUITARY RADIOTHERAPY: AN EFFECTIVE SECOND LINE TREATMENT FOR PAEDIATRIC CUSHING'S DISEASE}

H.L. Storr, P.N. Plowman, P.V. Carroll, I. François, G. Krassas, G.M. Besser, A.B. Grossman, M.O. Savage. Depts of Endocrinology and Radiotherapy, St Bartholomew's; Royal London School of Medicine and Dentistry, London ECIA 7BE; Dept. of Paediatrics, University of Leuven, Belgium; and Dept. of Endocrinology, Pagania Hospital, Thessaloniki, Greece

Transsphenoidal surgery (TSS) is considered first-line treatment for Cushing's disease (CD). Options for treatment of post-operative hypercortisolaemia are pituitary radiotherapy (RT), repeat TSS or bilateral adrenalectomy. During 1983-2001, we treated 18 patients, age range 6.4-17.8 yrs, with CD: all underwent TSS and 11 were cured (post-operative serum cortisol $<50 \mathrm{nmol} / \mathrm{l})$. Seven $(39 \%$ ) had $09.00 \mathrm{~h}$ serum cortisol levels of $269-900 \mathrm{nmol} / \mathrm{l}$ during the immediate post-operative period (2-20 days), indicating lack of cure. These patients $(6$ males, mean age $11.7 \mathrm{yrs}$, range $6.4-17.8 \mathrm{yrs} ; 4$ prepubertal, 3 pubertal) received external beam pituitary RT, using a $6-M V$ linear accelerator, with a total dose of 4500 cGy in 25 fractions over 35 days. Hypercortisolaemia was controlled with metyrapone \pm aminoglutethimide/o' $p^{\prime} D D D(n=3)$ or ketoconazole $(n=4)$. The mean interval between RT and "cure", ie a mean serum cortisol on a day curve of $<150 \mathrm{nmol} / \mathrm{l}$, was $0.95 \mathrm{yrs}(0.25-2.86 \mathrm{yrs})$. Recovery of normal pituitary-adrenal function (mean cortisol $150-300 \mathrm{nmol} / \mathrm{l}$ ) occurred at a mean of $1.16 \mathrm{yrs}(0.40-2.86 \mathrm{yrs})$ post-RT. At 2 yrs postRT, puberty occurred early ( $9.8 \mathrm{yrs})$ in one male patient. Growth hormone $(\mathrm{GH})$ secretion was assessed at $0.6-2.5 y$ rs post-RT in all patients: 6 had GH deficiency (subnormal peak GH on glucagon/ insulin, range <1-17.9 $\mathrm{mU} / \mathrm{l})$. All 7 patients received hGH replacement. Follow-up of pituitary function 7.6 and 9.5 yrs post-RT in 2 patients showed normal gonadotrophin secretion and recovery of GH peak response to 29.7 and $19.2 \mathrm{mU} / \mathrm{L}$. In conclusion, pituitary RT is an effective and relatively rapid-onset treatment for paediatric $C D$ following surgical failure. GH deficiency occurred in $86 \%$ patients, but long-term follow-up suggests recovery of $\mathrm{GH}$ secretion and preservation of other anterior pituitary function.

\section{G6 IS THE GNRH TEST A PREDICTOR OF NEUROLOGICAL LESION IN CENTRAL PRECOCIOUS PUBERTY IN GIRLS?}

S.M. Ng, D. Cody, Y. Kumar Y, C.S. Smith, M. Didi. Department of Endocrinology, Alder Hey Children's Hospital, Liverpool

Aims: To assess the value of gonadotrophin releasing hormone $(\mathrm{GnRH})$ stimulation test in identifying intracranial abnormality in central precocious puberty (CPP) in girls presenting without clinical features of underlying disease

Methods: A retrospective study of all girls diagnosed with CPP at Alder Hey Children's Hospital over 15 years. We included in this study, girls who had no clinical features to suggest underlying disease at presentation. They underwent cranial MRI to exclude intracranial causes. They were not receiving any treatment. The results of the $\mathrm{GnRH}$ stimulation test was assessed in relation to the following: basal $\mathrm{LH}$ and FSH values, peak $\mathrm{LH}$ and FSH values, LH/FSH peak ratios, peak $\mathrm{LH}$ /basal $\mathrm{LH}$ ratios and peak $\mathrm{FSH}$ /basal $\mathrm{FSH}$ ratios at presentation. Comparison was made between the girls with and without underlying intracranial lesions with respect to the above parameters.

Results: Mean age at onset of puberty was 6.2 years (range 0.5 to 8.0$)$ Intracranial abnormalities were present in $10(15 \%)$ patients, while 57 girls $(85 \%)$ had no intracranial abnormalities. No significant difference was shown between girls with intracranial abnormality and girls without intracranial abnormality in the following: basal LH or FSH values, peak $\mathrm{LH}$ or FSH values, $\mathrm{LH} / \mathrm{FSH}$ peak ratios, peak $\mathrm{LH} /$ basal $\mathrm{LH}$ ratios or peak $\mathrm{FSH} /$ basal FSH ratio

Conclusion: The GnRH stimulation test does not identify those with underlying intracranial abnormality in girls presenting with CPP without clinical features to suggest underlying disease.

\section{G7 FAMILIAL DYSALBUMINAEMIC HYPERTHYROXINAEMIA (FDH), A THYROID TRAP!}

P. Desai, A. Lipscomb. Paediatric Department, St John's Hospital, Wood Street, Chelmsford, CM2 9BG

Thyroid function screen is one of the most frequently requested endocrine investigations. We describe three patients here with $\mathrm{FDH}$ who were erroneously treated for thyrotoxicosis.

Three patients, (Chart 1) one of them adult, presented with biochemically raised free T4 levels whilst clinically euthyroid, with no goitre. The thyroid function tests were done for symptoms of poor weight gain (Case 1\& 2), tiredness and tachycardia (Case 3). All the patients received carbimazole for a variable period and two patients (case 1 and 3) further received "Block and replacement" with thyroxine to try and control the rising TSH.

All patients received inappropriate treatment before the clinician realised that the diagnosis was in question and they were investigated further.

The results point the diagnosis to be FDH. They remained well off treatment.

$\mathrm{FDH}$ is characterised by strikingly elevated total $\mathrm{T} 4$ values and free T4 values, but normal serum TSH levels and are clinically euthyroid. This is due to enhanced T4 binding by abnormal serum albumin. FDH is an autosomal dominant inherited condition, first described in 1979 by Henneman and Lee.

We have described the cases above to highlight the fact that these patients are mistakenly thought to be thyrotoxic and subjected to treatment, obviously unnecessary and potentially harmful. This emphasises the need for caution in basing a diagnosis of thyrotoxicosis on a raised serum thyroxine concentration obtained in a screening test without awareness of conditions causing euthyroid hyperthyroxinaemia.

\begin{tabular}{lllllll|}
\multicolumn{6}{l}{ Abstract G7, Table 1} \\
\hline & Age & TSH & TotalT4 & FT4 & FT4 onRx & Rx Duration \\
\hline Case1 & 8 yr & 2.5 & 248 & 31.5 & 62.4 & 2 yrs \\
Case2 & 7 yr & 4.0 & 238 & 43.9 & 40.4 & 6 Month \\
Case3 & $33 y r$ & 1.27 & 196 & 44.4 & 40.8 & $1.3 y r$. \\
\hline
\end{tabular}

\section{G8 DRIED BLOOD SPOTS TO SCREEN THYROID FUNCTION IN CHILDREN WITH DIABETES}

K.M. Bailey, J.M. Anderson, S.K.Hall'. New Cross Hospital, Wolverhampton; 'Birmingham Children's Hospital

Insulin Dependant Diabetes is a common condition in children. It's association with autoimmune thyroid disease, in particular Hashimoto's Thyroiditis, is well documented with an incidence of $1-5 \%$. Thyroid stimulating hormone (TSH) measured on dried blood spot (DBS) is routinely used for screening neonates for hypothyroidism, but not established outside this group due to differences in haematocrit (DBS measures TSH on whole blood). Children with diabetes dislike venepuncture, use of DBS sampling could improve the adherence with surveillance of TSH in this group.

\begin{tabular}{llll} 
Abstract & G7, Table 2 & & \\
& $\begin{array}{l}\text { FT4 } \\
\text { (equilibrium dialysis }\end{array}$ & $\begin{array}{l}\text { Antibodies } \\
\text { T4/T3 }\end{array}$ & FDH screen \\
\hline Case 1 & 23.4 (Normal) & Negative & Positive \\
Case 2 & 19.6 & Negative & Positive \\
Case 3 & 18.6 & Negative & Positive \\
\hline
\end{tabular}


Aim: To evaluate the use of DBS TSH outside the neonatal period. To identify a sensitive and specific value, above which a serum thyroid function test should be performed. This will provide an acceptable method of surveillance, for children with diabetes in clinical practice, avoiding venepuncture in most cases.

Method: Local Ethics Committee approval and informed consent were given. Children attending a paediatric diabetic clinic had simultaneous finger prick $\mathrm{HbAlc}$, DBS, and serum TSH.

Result: Samples were collected from 64 children with diabetes. Two samples with elevated TSH were correctly identified by DBS. Analysis was by exact methods. For a DBS value of $3 \mathrm{mU} / \mathrm{L}$ or greater, $100 \%$ specificity has a $95 \%$ confidence interval $0.95-1.0$. Sensitivity had a wide confidence interval (0.22-1.0) due to the limited numbers with elevated TSH

Conclusion:This initial sample demonstrates that DBS TSH accurately reflects serum TSH for surveillance in this population, despite the limitation of few elevated TSH results. A cut off value of $3 \mathrm{mU} / \mathrm{L}$ identified all elevated TSH results, with $100 \%$ specificity for those in the normal range. DBS sampling for surveillance of TSH will enhance the care of children with diabetes.

\section{G9 INSULIN LISPRO: ITS ROLE IN REDUCING NOCTURNAL HYPOGLYCAEMIA IN YOUNG CHILDREN WITH TIDM}

M.E. Ford-Adams', E.J. Moore', N.P. Murphy', J.A. Edge', R.M. Williams², K.K. Ong ${ }^{2}$. Department of Paediatrics, University of Oxford; ${ }^{2}$ Department of Paediatrics, University of Cambridge

Aims: Long duration of action of soluble insulin given in the evening may contribute to the high prevalence of nocturnal hypoglycaemia seen in young children with TIDM. We examined whether replacing soluble insulin with lispro reduces this.

Methods: Open cross-over study comparing lispro versus soluble insulin in 23 (16 boys) prepubertal children, mean age 9.4yrs (range 7-1 lyrs) with TIDM on three injections/day; long-acting isophane insulin remained identical. At the end of each 4 -month treatment arm, an overnight 15-min venous sampled blood glucose (BG) profile was performed and $\mathrm{HbAl} \mathrm{c}$ was determined.

Results: Despite similar BG pre-tea (lispro vs. soluble: $6.5 \pm 1.0$ vs. $7.1 \pm 1.1 \mathrm{mmol} / \mathrm{l}, \mathrm{p}=0.5)$, BG was lower on lispro between 18:00-22:00 (AUC: $p=0.03$ ). In contrast, between 22:00-04:00 BG tended to be higher on lispro (AUC: $p=0.3$ ), and the prevalence of low BG (<3.5 mmol/l) was lower on lispro ( $8 \%)$ than on soluble insulin (13\%, $p=0.01)$. Between 04:00-07:00 BG and low BG prevalence were similar on each insulin (fasting BG: $6.1 \pm 0.8$ vs. $6.3 \pm 0.9 \mathrm{mmol} / \mathrm{l}$, $\mathrm{p}=0.8$ ). There was no difference in $\mathrm{HbAlc}$ (lispro vs. soluble: 8.6 vs. $8.7 \%, p=0.3)$. At the end of the study $79 \%$ of the children chose to continue with lispro.

Conclusions: Shorter duration of action of insulin lispro given before the evening meal may reduce the prevalence of early nocturnal hypoglycaemia without compromising $\mathrm{HbAlc}$ in young children with TIDM

\section{G10 INFLUENCE OF SIZE AT BIRTH AND POSTNATAL CATCH-UP GROWTH ON FASTING INSULIN LEVELS AND POST-ORAL GLUCOSE INSULIN RESPONSE IN 8YR OLD CHILDREN}

K.K.Ong', C. Petry', A. Watts', the ALSPAC team², J. Golding², D.B Dunger'. 'Department of Paediatrics, University of Cambridge; ${ }^{2}$ Unit of Perinatal and Paediatric Epidemiology, University of Bristol

Introduction: Thinness at birth has been associated with reduced insulin sensitivity in adult studies. In the Avon Longitudinal Study of Parents and Children (ALSPAC) birth cohort, thinness at birth is related to fetal growth restraint, and is usually followed by rapid ("catch-up") postnatal weight gain and increased body mass index (BMI) and adiposity at $5 y$ rs. We therefore examined the effects of thinness at birth and postnatal catch-up growth on insulin sensitivity and post-oral glucose (post-OGT) insulin response at $8 y$ rs.

Methods: In 852 children (456 male) with previous body measurements at birth and $3 y$ rs, insulin and glucose levels were measured in fasting and 30-mins post-OGT venous blood samples. The homeostasis model was used to estimate insulin sensitivity from fasting insulin and glucose levels, and 30-mins post-OGT insulin response $\left(\mathrm{I}_{30}\right)$ was corrected for 30-mins glucose levels $\left(G_{30}\right)\left[{ }^{\prime \prime} \mathrm{CIR}_{30}{ }^{\prime \prime}=\mathrm{I}_{30} /\left(\mathrm{G}_{30} \times\left(\mathrm{G}_{30}\right.\right.\right.$ $3.9 \mathrm{mmol} / \mathrm{l}$ ]. Change in weight SD score $0-3 y r s$, allowing for sex and gestational age, was used to define postnatal "catch-up" growth (0-3yrs gain in weight $>0.67$ SDS) "catch-down" (<-0.67 SDS) and "non-changers" (>-0.67 and <0.67 SDS).
Results: Fasting insulin levels \& $\mathrm{CIR}_{30}$ were positively correlated $(r=0.26, \quad p<0.0005)$, and both were related to current weight $(r=0.26, p<0.0005 \& r=0.27, p<0.0005), B M I(r=0.25, p<0.0005$; $r=0.27, p<0.0005)$ and waist circumference $(r=0.24, p<0.0005$ \& $r=0.25, p<0.0005$ ). Postnatal catch-up growth 0-3yrs was associated with higher fasting insulin levels $(p<0.005)$, reduced insulin sensitivity $(p<0.005)$ and increased $\mathrm{CIR}_{30}(p<0.005) \mathrm{com}$ pared to other children. A weak relationship between thinness at birth (ponderal index) and reduced insulin sensitivity was only seen after allowing for current $\mathrm{BMI}$ in children who showed postnatal catch-up or catch-down growth $(r=0.11, p<0.05)$. Independent of postnatal growth rate, thinness at birth was also associated with reduced $\mathrm{CIR}_{30}$ $(p<0.005)$

Conclusions: Associations between small size at birth and reduced insulin sensitivity may depend on subsequent postnatal catch-up growth and obesity risk. However, independent of postnatal growth rate and obesity risk, thinness at birth may be related to a reduced insulin response to glucose.

\section{G1 1 FIVE YEAR-OLD GIRLS ARE INTRINSICALLY MORE INSULIN RESISTANT THAN BOYS (THE EARLYBIRD STUDY)}

K.M. Mallam, M.J. Murphy', B.S. Metcalf, A.N. Jeffery, L.D. Voss, T.J. Wilkin. University Medicine and 'Combined Laboratories, Level 7, Derriford Hospital, Plymouth, PL6 8DH

Introduction: Type 2 diabetes (T2D) and metabolic syndrome are the result of insulin resistance (IR), and their incidence is rising. Previously known as "adult-onset" diabetes, T2D is now occurring in children with a strong, but unexplained, female predominance.

Aim: To explain the female predominance in early-onset T2D.

Methods: The EarlyBird Study is a non-intervention, prospective cohort study of randomly selected healthy school entrants in the SW of England. Measures include IR by HOMA on fasting serum, anthropometry including BMI and subcutaneous fat by skinfolds (SF), physical activity (PA) using CSA accelerometers and the metabolic correlates of IR.

Results: Baseline data were obtained on 307 children, mean age 4.9 years. Girls were $27 \%$ more insulin resistant than boys $(p<0.001)$ When corrected for SF and total PA, the remaining difference was still $19 \%(p<0.05)$. SF was $23 \%$ higher in girls $(p<0.001)$, but BMI only $3 \%$ higher $(p=0.01)$. Waist circumferences were no different, though the hips of girls were broader. SF had the greatest effect on gender difference in IR $(-9 \%)$. Boys undertook more PA than girls $(p=0.01)$ but the difference had minimal impact $(+1 \%)$ on IR. Girls had higher triglycerides $(p<0.01)$, lower HDL $(p<0.05)$ and lower SHBG $(p<0.05)$ than boys.

Conclusions: Girls are intrinsically more insulin resistant than boys, even when corrected for body fat mass and PA differences. The observation may explain the female predominance of type 2 diabetes reported in children.

\section{G12 SURVEY OF NON-TYPE 1 DIABETES SHOWS UK TYPE 2 DIABETES PREVALENCE $0.4 \%$ DISTINCT FROM MODY, AND ASSOCIATED WITH OVERWEIGHT, PUBERTY,} FEMALE SEX AND ETHNIC MINORITY STATUS

T. Barrett,, S. Ehtisham ${ }^{1,2}$, A. Hattersley ${ }^{3}$. Birmingham Children's Hospital'; University of Birmingham ${ }^{2}$; University of Exeter ${ }^{3}$

Most children with diabetes have type 1 (insulin deficient); however, we have reported type 2, as defined by evidence of insulin resistance; there is diagnostic confusion with genetic subtypes such as Maturity Onset Diabetes of the Young (MODY), and the prevalence and characteristics of non-type 1 diabetes in UK children is unknown. We aimed to characterise and determine the prevalence of non-type diabetes. We undertook a cross-sectional questionnaire survey of all paediatric diabetes centres during 2000 , and collected data on mode of presentation, clinical characteristics, and investigations. 172/228 centres responded $(75 \%)$, and manage 15,255 diabetics under 16 years. They provided data on 113 children with non-type 1 diabetes $(0.8 \%)$. Of these, 25 had type 2 diabetes $(0.2 \%)$, of whom 8 presented in $2000(0.4 \%)$. 24 had MODY, 45 had diabetes associated with a variety of other conditions, and 19 unknown. Type 2 diabetes and MODY showed different clinical characteristics. Type $2 \vee$ MODY: age of presentation 12.8 years (3.6-15.9) (median(range)) v 10.75 yrs $(3.1-14.8)$, NS; female $(75 \%$ v $64 \%$, NS) ethnic minorities $(64 \% \vee 0 \%, P<0.01)$, family history of diabetes $(80 \% \vee$ 
$100 \%, \mathrm{NS})$, overweight or obese $(96 \%$ v $25 \%, \mathrm{p}<0.01)$. At presentation most Type 2 children were asymptomatic, but 4 had ketonuria. Type 2 diabetes is disproportionately affecting ethnic minority, pubertal, overweight girls with a family history of diabetes. It is distinguished from MODY by the presence of insulin resistance.

\section{G13 MOLECULAR GENETICS OF CONGENITAL ADRENAL HYPERPLASIA (21 HYDROXYLASE DEFICIENCY): IMPLICATIONS FOR BEHAVIOURAL MASCULINISATION IN GIRLS}

C.M. Hall', J. Jones', H.F.L. Meyer-Bahlburg', C. Dolezal'2, M. Coleman $^{3}$, D.A. Price', P.E. Clayton'. 'Royal Manchester Children's Hospital, UK; ${ }^{2}$ Department of Psychiatry; Columbia University, USA; ${ }^{3}$ Department of Genetics; St Mary's Hospital, Manchester, UK

Females with congenital adrenal hyperplasia (CAH) are more masculine and less feminine than their peers and their behaviour is thought to result from exposure of the fetal brain to high concentrations of androgens. Using 2 highly sensitive measures: the Child Game Participation Questionnaire [CGPQ] (Meyer-Bahlburg et al, 1994) and the Child Behaviour and Attitude Questionnaire [CBAQ] (MeyerBahlburg et al, 1994) a group of $24 \mathrm{CAH}$ girls and 25 diabetic (D) girls aged 3-12 years from Manchester were compared and we have reported that $\mathrm{CAH}$ girls were significantly more masculine and less feminine than $D$ girls ( $p<0.000$ for both) and that the $D$ girls were not significantly different from USA community norms (Hall et al, 2001). Several studies have demonstrated a relationship between genotype and biochemical phenotype in $\mathrm{CAH}$, and the aim of this study was to assess whether such a relationship exits between genotype and behavioural masculinisation.

In Manchester, mutation detection based on allele specific PCR is used for genetic diagnosis of CAH. Mutations screened include cyp2 1 deletion/large gene conversion and the following pseudogenederived mutations: $655 \mathrm{~A} / \mathrm{C}>\mathrm{G}, 2108 \mathrm{C}>\mathrm{T}, 999 \mathrm{~T}>\mathrm{A}$. The latter 3 mutations are reported to cause similar virilisation and salt loss to cyp 21 deletion. The genotypes of the 24 girls studied (23 salt wasters) were categorised thus: $(1)=$ homozygous cyp 21 deletion $[n=8],(2)$ cyp2 1 deletion/ (655A/C>G OR 2108 C >T OR 999 T>A) [n=8], (3) homozygous (655A/C>G OR $2108 \mathrm{C}>\mathrm{T}$ OR $999 \mathrm{~T}>\mathrm{A})[\mathrm{n}=3],(4)$ heterozygous combination of $(655 \mathrm{~A} / \mathrm{C}>\mathrm{G}, 2108 \mathrm{C}>\mathrm{T}$, $999 \mathrm{~T}>\mathrm{A}$, undetected mutation) [n=5]

From the $C B A Q$ and $C G B Q$ an overall masculinity score $(M)$ was derived and regression analysis indicated that $27 \%$ of the variability in $M$ was predicted by the genotype ( $p=0.013)$ : mean $M$ was significantly lower in group $4(3.1)$ than in group $3(4.3)[p=0.01]$, group 2 (4.3) $[p=0.002]$ and group 1 (4) $[p=0.014]$. There was a positive correlation between Prader degree of virilisation at birth and M $\mathrm{r}=$ $0.4, p=0.05$ ). In conclusion, this study is the first to demonstrate a relationship between genotype and behavioural masculinisation in girls with $\mathrm{CAH}$ 